
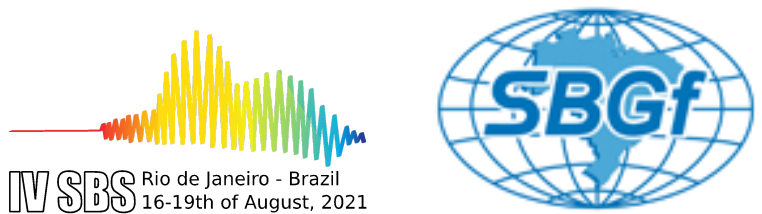

\title{
Investigating crustal structure under the Brazilian Shield with P-wave autocorrelations
} José Richarles Almeida Gomes, Jordi Julià Casas, UFRN/PPGG

Copyright 2021, SBGf - Sociedade Brasileira de Geofísica.

This paper was prepared for presentation during the $17^{\text {th }}$ International Congress of the Brazilian Geophysical Society held in Rio de Janeiro, Brazil, $16-19$ August 2021.

Contents of this paper were reviewed by the Technical Committee of the $17^{\text {th }}$ International Congress of the Brazilian Geophysical Society and do not necessarily represent any position of the SBGf, its officers or members. Electronic reproduction or storage of any part of this paper for commercial purposes without the written consent of the Brazilian Geophysical Society is prohibited.

\begin{abstract}
The basic principle of seismic interferometry is to generate a new structural response from the cross-correlation between two seismic traces. In the particular case in which the correlation is between a seismic trace and itself, the operation is called an autocorrelation. It has already been shown that the autocorrelation of the transmission response at a given receiver is equivalent to the reflectivity response under that same receiver. In the last decade, the autocorrelation functions (ACFs) have been applied to seismic noise records to extract structural information on the crust and upper mantle. Although it does not depend on the occurrence of earthquakes, this approach presents as a disadvantage the need for a large volume of data to converge to a stable reflectivity response. Alternatively, it has been proposed that the autocorrelation of the P-wave coda of teleseismic events (i.e., distant events) might be also utilized to recover the structural response. In this new approach, it is possible to obtain comparable results with a smaller amount of data. Furthermore, the radial ACFs are similar to P-wave receiver functions, while the vertical ACFs display the P-wave reverberation (PPmP) that is missing in P-wave receiver functions (PRFs). Consequently, ACFs can provide information about both the P-wave velocity structure. Taking this into account, this work aims to conduct a new crustal investigation of the Brazilian Shield using the teleseismic $\mathrm{P}$ wave autocorrelation functions. The goal is to develop new estimates of crustal thickness and compare to previous estimates from receiver function studies. To this end, data from the 93 broadband stations making up the Brazilian Seismographic Network (RSBR) was considered. Teleseismic sources were selected according to two criteria: a minimum magnitude of $5.5 \mathrm{mb}$, to guarantee a good signal-to-noise ratio; and epicentral distances of $30^{\circ} \leq \Delta \leq 90^{\circ}$, to avoid mantle triplications and shadow zones. We also considered distances above $120^{\circ}$ (PKP and PKIKP phases) to augment our data set. The selected recordings were cut 10 seconds before and 110 seconds after the theoretical $P$ wave arrival time and, after pre-processing and spectral whitening, autocorrelation in the frequency domain and phase-weighted stack was applied. The resulting ACFs allow for identification of the P-to-S conversions and multiple reverberations between the surface and the base of the crust, showing promise for the determination of independent $P$ and $S$ wave velocity structure for the Brazilian Shield.
\end{abstract}

